\title{
Óptimos económicos en la cría del cerdo pelón mexicano: propuesta de integración para cadena productiva
}

\section{Economic optimals in production of mexican hairless pig: integration proposal for productive chain}

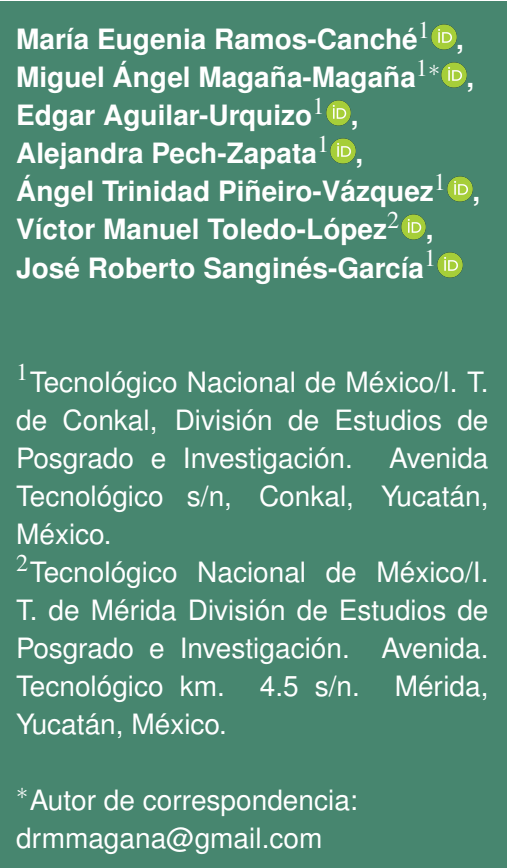

Artículo científico

Recibido: 25 de mayo de 2019

Aceptado: 26 de noviembre de 2019

Como citar: Ramos-Canché ME, Magaña-Magaña MA, Aguilar-Urquizo E, Pech-Zapata A, Piñeiro-Vázquez AT, Toledo-López VM, SanginésGarcía JR (2020) Óptimos económicos en la cría del cerdo pelón mexicano: propuesta de integración para cadena productiva. Ecosistemas y Recursos Agropecuarios 7(1): e2302. DOI: 10.19136/era.a7n1.2302

RESUMEN. El objetivo del estudio fue determinar la cantidad óptima de alimento y peso del cerdo pelón mexicano (CPM) que se asocie a la máxima ganancia. Se utilizó la información de 22 hembras de CPM con un peso inicial de $5.07 \pm 1.43 \mathrm{~kg}$ y final de $95.62 \pm 12.09 \mathrm{~kg}$; el ciclo de engorda se dividió en cuatro etapas. Para determinar los óptimos de consumo de alimento, peso y edad de venta del animal se empleó una función de producción representada por un modelo polinomial cuadrático; se consideraron precios de venta de $30.00,35.00,40.00$ y $\$ 45.00$ por $\mathrm{kg}$ de PV con alimentación $100 \%$ alimento balanceado y otro con restricción de $18 \%$. En las etapas I y II se registró la mayor eficiencia alimenticia con 0.37 y 0.30 $\mathrm{kg}$ de ganancia por $\mathrm{kg}$ de alimento. El producto marginal promedio fue decreciente (0.24, $0.23,0.21$ y 0.15 por $\mathrm{kg}$ de alimento para las etapas I, II, III y IV). El peso óptimo de mercado se incrementó en el mismo sentido que el precio de venta del animal siendo: 15.50 a $75.00 \mathrm{~kg}$ en la alimentación de $100 \%$ alimento balanceado y 46.70 a $86.80 \mathrm{~kg}$ en la restringida. Debido al bajo valor de los parámetros obtenidos hacen necesaria la integración de la actividad primaria como parte de la cadena productiva con la finalidad de obtener la máxima ganancia y conservación de dicho recurso zoogenético.

Palabras clave: Función de producción, eficiencia alimenticia, producto marginal, peso óptimo, rentabilidad.

ABSTRACT. The objective was to determine the optimum amount of food and weight of the hairless mexican pig (HMP) that is associated with the maximum gain. It were used information of 22 females of (HMP), initial weight $5.07 \pm 1.43$ $\mathrm{kg}$ and final weight $95.62 \pm 12.09 \mathrm{~kg}$; the production cycle was divided into four stages. To determine the optimal food intake, weight and age of sale of the animal, a production function represented by a quadratic polynomial model was used; it was considered selling prices of $30.00,35.00,40.00$ and 45.00 mexican pesos / $\mathrm{kg}$ with $100 \%$ balanced feed and another with $18 \%$ restriction. In stage I and II the highest food efficiency was registered with 0.37 and $0.30 \mathrm{~kg}$ of gain per $\mathrm{kg}$ of feed. The average marginal product was decreasing $(0.24,0.23,0.21$ and 0.15 per $\mathrm{kg}$ of food for each stage I, II, III and IV). The optimum market weight was increased in the same sense as the sale price of the animal being: 15.50 to $75.00 \mathrm{~kg}$ in the feed of $100 \%$ balanced feed and 46.70 to $86.80 \mathrm{~kg}$ in the restricted feed. Due to the low value of the parameters obtained, it is necessary to integrate the primary activity as part of the productive chain in order to obtain the maximum gain and conservation of said animal genetic resource.

Key words: Production function, food efficiency, marginal product, optimal weight, profitability. 


\section{INTRODUCCIÓN}

La producción de alimentos inocuos de origen animal, libres de antibióticos y agroquímicos, representa un reto para los sistemas de producción intensivos, a pesar de los beneficios potenciales para la salud humana (Mie et al. 2017), por tal razón, la tendencia actual de demanda de este tipo de alimentos (orgánicos) se ha incrementado en los últimos años. Por otra parte, la producción orgánica de carne de cerdo tiene beneficios ambientales y de bienestar animal (Grunert et al. 2018) ya que se realiza en granjas semintensivas como se observa en las explotaciones rurales y de traspatio del cerdo pelón mexicano (CPM). Al respecto, se ha observado que el uso de la fibra dietética en cerdas gestantes mejora el bienestar animal al reducirse las conductas estereotipadas (Oelke et al. 2018); por lo que el uso del follaje de arbóreas con potencial forrajero representa un aporte de proteína de buena calidad y de fibra con bajo contenido de lignina, lo que puede ayudar a reducir los costos de producción por concepto de alimentación; pero la presencia de metabolitos secundarios podría limitar su inclusión en la dieta (Régnier et al. 2013).

EI CPM es una variante genética de los cerdos criollos de América Latina, los cuales se originaron de los cerdos ibéricos (Sus mediterraneus) y poseen una gran variabilidad genética (Su et al. 2014). Este genotipo pertenece a un animal longilíneo con tendencia a la acumulación de grasa en la canal, dado que a partir de los $70.00 \mathrm{~kg}$ el aumento en el peso corporal se debe principalmente a la síntesis de tejido adiposo (Méndez et al. 2002), el cual es mayor con respecto a cerdos magros (Camacho-Rea et al. 2008). Estas características son similares a las de los cerdos criollos del Caribe, que tienen un crecimiento lento y pobre rendimiento en canal (Renaudeau y Mourot 2007). Con respecto al cerdo ibérico, Nieto et al. (2014) demostraron que con base en la composición química del animal completo y el crecimiento relativo de los tejidos de la canal, éstos no se ajustan a los modelos de crecimiento de los cerdos magros y de los genotipos convencionales, descendientes de los cerdos célticos (Sus scrofa).
La demanda del CPM es inferior con respecto a la de los cerdos magros, por dos razones principales, la primera es el elevado contenido de grasa en la canal y la otra es el bajo rendimiento de carne magra, por lo que su precio de venta en pie es inferior de 30 a 40\% (Méndez et al. 2002). Una alternativa para valorarlo es a través de la propuesta de nuevas estrategias de mercado, donde se aprovechen las cualidades de su carne, como son el alto contenido de grasa subcutánea e intramuscular y su perfil de ácidos grasos favorables para la elaboración de productos curados de alta calidad (Delgado et al. 2002, $a b)$; características que dependen de la genética del cerdo. Entre las ventajas que ofrece la carne del CPM está la lenta deshidratación de los productos durante el curado, lo que permite largos periodos de industrialización y mayor calidad (Serra et al. 1998).

A pesar de las cualidades que presenta la carne del CPM, éstas no han sido reconocidas o valoradas en el mercado debido a la falta de investigación y difusión de resultados. Por ejemplo, no se cuenta con información de su comportamiento productivo, el uso óptimo de insumos, prácticas de manejo fuera del sistema tradicional, entre otros, que permitan mejorar la producción y rentabilidad; situación que lo deja en desventaja frente a otras razas porcinas (Delgado et al. 2002b). Por lo que es relevante conocer los factores que ayuden a determinar el óptimo económico en el proceso de la engorda, con lo cual se aprovecharía mejor este recurso zoogenético. Siendo las razas locales de cerdos una reserva de alto valor genético, las cuales pueden usarse para recuperar las propiedades organolépticas de la carne, que se han perdido a causa de los programas de mejoramiento genético que se enfocan en la mejora cuantitativa de la producción (Pugliese y Sirtori 2012).

Una alternativa viable de valoración y aprovechamiento sustentable de los recursos zoogenéticos con ventajas para la producción empresarial, es la integración de éstos a una cadena productiva y de valor (Peña et al. 2008). Así, el CPM tiene la posibilidad de integrarse a un sistema constituido para la comercialización de productos con valor agregado, ya que cuando el porcicultor envía por su cuenta los animales para abasto primero se enfrenta 
a los tablajeros que ofertan carne fresca en los mercados municipales, segundo a los introductores de animales al rastro $y$, tercero, con los mayoristas $y$ minoristas de carne en canal, quienes no valoran la calidad de la carne y sus características organolépticas, con lo cual la rentabilidad de la empresa es mínima o negativa. Ante lo expuesto anteriormente, y con el fin de contribuir con información técnica y económica del primer eslabón de la cadena productiva del CPM, el objetivo fue determinar la cantidad óptima de alimento y el peso del animal a la venta asociado con la máxima ganancia económica, para lo cual se consideraron diversos escenarios de precios de compra de alimento y de venta del animal destinado al abasto de la agroindustria procesadora de jamones y otros productos curados de alta calidad.

\section{MATERIALES Y MÉTODOS}

El trabajo se realizó en la unidad de Producción e Investigación Agropecuaria del Instituto Tecnológico de Conkal, Yucatán, ubicado en las siguientes coordenadas: $21^{\circ} 05^{\prime} \mathrm{LN}, 89^{\circ} 32^{\prime} \mathrm{LO}$; altura de $8 \mathrm{~m}$ sobre el nivel del mar; el clima es cálido subhúmedo con lluvias en verano (Aw0) de acuerdo con la clasificación de Köeppen (García 1981).

Se utilizó la información referente al cambio de peso vivo (PV) y consumo de alimento de 22 hembras de Cerdo Pelón Mexicano, que no cumplieron con las características fenotípicas para ser incorporadas dentro de la piara reproductora, por lo que se integraron a la engorda junto con los machos castrados. La edad inicial fue $33 \pm 3$ días, con un peso de $5.07 \pm 1.43 \mathrm{~kg}$; la engorda finalizó cuando éstas alcanzaron un peso promedio de $95.62 \pm 12.09 \mathrm{~kg}$. El manejo del lote experimental fue de acuerdo a la guía para el cuidado y uso de animales de laboratorio (NRC 2010), los cuales se alojaron en corrales elevados individuales $(2.00 \times 0.70 \mathrm{~m})$ con piso de rejilla, provistos de comedero y bebedero de chupón; el alimento se proporcionó a libre acceso, pesando lo ofrecido y rechazado para estimar el consumo por etapa. Se formularon cuatro dietas de acuerdo con las recomendaciones del NRC (2012), el experimento se dividió en cuatro etapas (Tabla 1), que comprendieron del destete al peso al sacrificio. Con excepción del alimento preiniciador, el resto se elaboró en la unidad de producción. Los animales se pesaron cada semana desde el inicio hasta el final del experimento.

Para la determinación de la eficiencia alimenticia, se utilizó la fórmula:

$$
\text { Eficiencia alimenticia }=\frac{G P}{C A}
$$

Dónde: $\mathrm{GP}=$ ganancia de peso $(\mathrm{kg})$ y $\mathrm{CA}=$ alimento consumido $(\mathrm{kg})$.

En la determinación de los óptimos económicos de consumo de alimento, peso y edad de venta del animal se utilizó la función de producción representada por un modelo polinomial cuadrático; considerando diferentes escenarios de precios de venta: $30.00,35.00,40.00$ y 45.00 pesos mexicanos por $\mathrm{kg}$ de PV, mientras que el precio del alimento fue el promedio ponderado estimado en las diferentes etapas de alimentación. El modelo de regresión lineal múltiple que representó la función de producción porcina, fue:

$$
P V=\beta_{0}+\beta_{1} \cdot C-\beta_{2} \cdot C^{2}+\varepsilon
$$

Dónde: $\mathrm{PV}=$ peso vivo del animal, $\mathrm{C}=$ consumo de alimento, $\beta=$ coeficiente de regresión para $i=0,1,2$, y $\varepsilon=$ término de error.

De acuerdo con la teoría económica (Kay 1986), el punto óptimo de producción $\left(\mathrm{PV}^{*}\right)$ asociado a la máxima ganancia se obtiene cuando el precio de venta del animal (Ppv) en el mercado competitivo se iguala al costo marginal (CMg). Así mismo, el costo marginal es igual al cociente entre el precio del alimento (Pal) y el producto marginal (PMg), representado por las siguientes fórmulas:

$$
P p v=C M g=\frac{P a l}{P M g}
$$

Dónde: $P M g=\frac{d P V}{d C}, \mathrm{y} \mathrm{d}=$ razón de cambio (derivada) El valor para el producto marginal fue:

$$
P M g=0.24582092+0.000294 C
$$


Tabla 1. Etapas de alimentación de los cerdos en función del peso.

\begin{tabular}{lccc}
\hline Etapa & $\begin{array}{c}\text { Peso de } \\
\text { las cerdas }\end{array}$ & $\begin{array}{c}\text { Contenido de } \mathrm{P} \\
\left(\mathrm{g} \mathrm{kg}^{-1} \text { de alimento }\right)\end{array}$ & $\begin{array}{c}\text { Contenido de EM } \\
\left(\mathrm{kcal} \mathrm{kg}^{-1} \text { de alimento }\right)\end{array}$ \\
\hline Preiniciación & Destete $(5.07)$ hasta $10.10 \mathrm{~kg}$ & 200.00 & 3400.00 \\
Iniciación & $10.00 \mathrm{a} 25.00 \mathrm{~kg}$ & 180.00 & 3200.00 \\
Crecimiento & 25.00 a $50.00 \mathrm{~kg}$ & 130.00 & 3300.00 \\
Finalización & $50.00 \mathrm{a}>100.00 \mathrm{~kg}$ & 115.00 & 3300.00 \\
\hline${ }^{*}$ Alimento comercial. & & &
\end{tabular}

\section{RESULTADOS}

El peso final de los animales que integraron la piara experimental fue $95.62 \pm 12.09 \mathrm{~kg}$ y la edad se ajustó a 280 días, con consumo de alimento total de $510.90 \mathrm{~kg}$ a partir del destete. Durante el periodo de engorda (252 días), a partir del destete, los animales ganaron un peso de $90.50 \mathrm{~kg}$, lo que equivale a una ganancia diaria promedio de $0.36 \mathrm{~kg}$ con consumo promedio diario de $2.03 \mathrm{~kg}$, y eficiencia de 0.18 (Tabla 2). No obstante, se observó una eficiencia diferencial en función de la etapa evaluada, siendo mayor en las primeras dos etapas con respecto a la últimas dos; pero éstas no impactaron de forma considerable la eficiencia final promedio, dado que el consumo acumulado de las dos primeras etapas fue $61.8 \mathrm{~kg}$ de alimento ( $13.76 \%$ del total), mientras que el de las dos últimas fue de $449.10 \mathrm{~kg}$.

En la etapa I se requirió $0.57 \mathrm{~kg}$ de alimento para producir $0.21 \mathrm{~kg} \mathrm{~d}^{-1}$, en esta etapa fue mayor el potencial de crecimiento de las cerdas, observándose ganancias significativas de peso; pero fue la etapa con menor duración y consumo. En la etapa II se observó una tasa de crecimiento menor con respecto a la primera, ya que los animales ganaron $0.39 \mathrm{~kg} \mathrm{~d}^{-1}$ de peso vivo por $1.31 \mathrm{~kg}$ de alimento.

En la etapa III la ganancia de peso por animal $\left(0.38 \mathrm{~kg} \mathrm{~d}^{-1}\right)$ fue ligeramente menor a la obtenida en la etapa II, en la que se requirió mayor consumo de alimento. Por su parte, la etapa de mayor tiempo de engorda fue la IV, ésta se consideró la menos eficiente; la ganancia de peso por animal fue de 0.37 $\mathrm{kg} \mathrm{d}^{-1}$ por $2.62 \mathrm{~kg}$ de alimento.

En la Figura 1 se presenta la relación que se establece entre peso vivo del animal (PV) y el consumo de alimento $(C)$, conocida también como función de producción, cuyos parámetros estimados se observan en la siguiente fórmula:

$$
P V=7.93907478+0.24582092 C-0.000147 C 2, \quad R 2=0.99772
$$

$$
\begin{array}{lll}
(0.50615) & (0.00546) & (1.1015 E-05) \quad \text { ANDEVA } p<0.0001
\end{array}
$$

El valor estimado de los parámetros en la función de producción permite analizar el comportamiento productivo de las cerdas, donde el consumo de alimento balanceado se consideró como el factor más significativo. Estos parámetros indican que la eficiencia alimenticia de los animales fue decreciente, debido a que éstos ganaron cada vez menos peso a medida que aumentó el consumo de alimento. Lo que se evidencía con el producto marginal promedio calculado con la fórmula anterior, siendo de 0.24, 0.23, 0.21 y 0.15 por $\mathrm{kg}$ de alimento para las etapas I, II, III y IV, respectivamente.

Para analizar los cambios de rentabilidad en la engorda del CPM, se consideran diferentes precios ponderados de alimento y precios de venta, mismos que permitieron determinar el peso y edad óptima de los animales que se transferirán al siguiente eslabón de la cadena productiva (Tabla 3). Estos escenarios son de importancia en la toma de decisiones respecto a las condiciones productivas y de mercado, por ejemplo el peso óptimo de venta puede variar según el nicho de mercado al que se quiera acceder. En el presente estudio se identificaron dos nichos relevantes, el primero fue el de canales de no más de $12.00 \mathrm{~kg} \mathrm{y}$, el segundo, el de la agroindustria que demandan animales finalizados para la elaboración de productos curados.

\section{DISCUSIÓN}

En las primeras dos etapas se registró la mayor eficiencia alimenticia con 0.37 y 0.30 , respectivamente. En la etapa I se observó el mayor potencial de crecimiento de las cerdas, con ganancias de peso 
Tabla 2. Etapa y costo de la alimentación del cerdo pelón mexicano.

\begin{tabular}{cccccc}
\hline $\begin{array}{c}\text { Etapa de } \\
\text { alimentación }\end{array}$ & $\begin{array}{c}\text { Duración de la } \\
\text { etapa (días) }\end{array}$ & $\begin{array}{c}\text { Edad del animal al } \\
\text { final de etapa (días) }\end{array}$ & $\begin{array}{c}\text { Peso del animal al } \\
\text { final de etapa }(\mathrm{kg})\end{array}$ & $\begin{array}{c}\text { Consumo de alimento } \\
\text { por etapa }(\mathrm{kg})\end{array}$ & $\begin{array}{c}\text { Costo del alimento } \\
\text { por etapa }(\$)\end{array}$ \\
\hline I & 28.00 & 56.00 & 11.00 & 16.10 & 244.80 \\
II & 35.00 & 91.00 & 24.50 & 45.70 & 410.70 \\
III & 70.00 & 161.00 & 51.30 & 137.40 & 973.40 \\
IV & 119.00 & 280.00 & 95.60 & 311.70 & 2027.50 \\
\hline
\end{tabular}

El peso del animal al inicio de la primera etapa fue de $5.07 \mathrm{~kg}$.

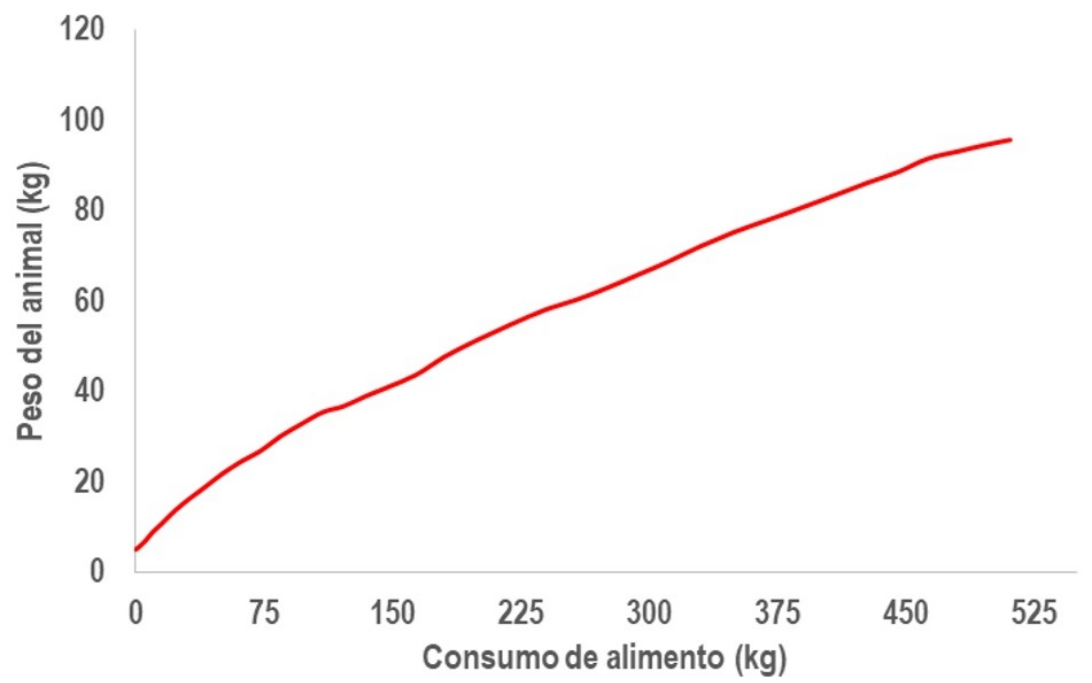

Figura 1. Función de producción en la engorda del cerdo pelón mexicano.

Tabla 3. Óptimo económico en la engorda del cerdo pelón mexicano.

\begin{tabular}{ccccc}
\hline $\begin{array}{c}\text { Precio ponderado del } \\
\text { alimento por } \mathrm{kg}(\$)\end{array}$ & $\begin{array}{c}\text { Precio de venta del } \\
\text { animal en } \mathrm{kg}(\$)\end{array}$ & $\begin{array}{c}\text { Consumo óptimo de } \\
\text { alimento en } \mathrm{kg}\end{array}$ & $\begin{array}{c}\text { Peso óptimo de venta } \\
\text { del animal en } \mathrm{kg}\end{array}$ & $\begin{array}{c}\text { Edad óptima } \\
\text { del animal (días) }\end{array}$ \\
\hline 6.50 & 45.00 & 344.80 & 75.20 & 218.00 \\
6.50 & 40.00 & 283.40 & 65.80 & 195.00 \\
6.50 & 35.00 & 204.40 & 52.10 & 162.00 \\
7.10 & 30.00 & 31.10 & 15.50 & 68.00 \\
& & & & \\
\hline 5.30 & 45.00 & 433.20 & 86.80 & 249.00 \\
5.30 & 40.00 & 382.90 & 80.50 & 232.00 \\
5.30 & 35.00 & 317.80 & 71.20 & 208.00 \\
5.80 & 30.00 & 176.30 & 46.70 & 150.00 \\
\hline
\end{tabular}

significativas, debido a que el alimento proporcionado fue de mayor calidad nutritiva, con elevado porcentaje de lisina y proteína, los cuales son requeridos en éste período de crecimiento en las diferentes razas porcinas (NRC 2012). Esta etapa es la de menor duración y por consiguiente el consumo de alimento es menor, con mayor costo. Al respecto, Rebollar et al (2007) al evaluar el óptimo económico en una granja porcina reporta comportamientos similares, con producto marginal máximo en la etapa de iniciación, la cual corresponde a la etapa I del presente trabajo, siendo la más eficiente debido a la velocidad de transformar el insumo variable en producto total.

Por otra parte, en la etapa III la ganancia de peso por animal $\left(0.38 \mathrm{~kg} \mathrm{días}^{-1}\right)$ fue similar a la obtenida en la etapa II, pero requirieron mayor consumo de alimento, con eficiencia menor ( 0.20 vs 0.30), y la etapa de mayor tiempo de engorda fue la IV (Tabla 2), con prácticamente el doble de la duración de las etapas II y III, indicando un crecimiento lento a partir de los $50 \mathrm{~kg}$, etapa en la cual los animales alcanzaron la madurez y es la menos eficiente, con 
ganancia de peso de $0.37 \mathrm{~kg} \mathrm{~d}^{-1}$, con eficiencia de 0.14 .

En la función de producción, el consumo de alimento balanceado se consideró como el factor más significativo, lo que explica el comportamiento productivo de las cerdas (Figura 1). La eficiencia alimenticia de los animales fue decreciente, debido a que ganaron menos peso a medida que aumentó el consumo de alimento. Lo que se evidencia con el producto marginal promedio calculado, de $0.24,0.23$, 0.21 y 0.15 por $\mathrm{kg}$ de alimento en las etapas I, II, III y IV. Lo que indica el cambio que experimenta el peso del animal con relación al cambio en la cantidad de alimento consumido, es decir el cociente de ambos muestra la pendiente de la función de producción; siendo positivo cuando los valores de la función de producción tiende a su punto máximo y negativo, cuando los valores de la función disminuyen después de alcanzar dicho punto. La teoría económica indica que el valor decreciente del producto marginal estimado por etapa de alimentación muestra que la engorda se encuentra en la fase II de la función de producción (Pindyck y Rubinnfeld 2009), donde se presenta la máxima ganancia, según los precios del alimento y de venta del animal.

Con el fin de analizar diversos escenarios de rentabilidad en la engorda de CPM, se consideran diferentes precios ponderados de alimento y precios de venta (Tabla 3). El primer escenario es el óptimo económico que se asocia al segmento del mercado donde se ofertan canales de animales pequeños, determinándose para éste un peso óptimo de venta de $15.50 \mathrm{~kg}$ por animal, que corresponde a la etapa II, donde la eficiencia alimenticia no es la mayor de la engorda si se compara con la primera etapa. El precio por kilogramo de animal vivo, que se relaciona con el óptimo económico en este escenario es de $\$ 30.00$; con margen bruto sobre los costos de alimentación de $\$ 242.40$ por animal, con el $100 \%$ de alimento balanceado. En la determinación del óptimo económico en la etapa II se consideró el precio de venta y el costo marginal de producción, propio del enfoque basado en el análisis marginal, por lo que en la contabilidad de costos se debe de incluir el valor de adquisición del animal al destete. Por lo que el valor de venta de un animal compende del ingreso marginal obtenido (\$312.90) más el valor de su adquisición al destete; por lo que el valor de venta a salida a granja de un animal con el peso óptimo es de aproximadamente $\$ 1200.00$; dado que en general en esta etapa los animales se comercializan por pieza y no por peso.

Los siguientes escenarios de simulación corresponden a la etapa IV de engorda de CPM, debido a que en ésta el peso que alcanzan los animales son los requeridos para la elaboración de productos curados de alta calidad (Lopez-Bote et al. 2000). Por ejemplo, el contenido de grasa subcutánea e intramuscular, principalmente en las piernas y paletas, le confieren características deseables como materia prima para la elaboración de productos derivados de la carne de cerdo; que según su peso pueden utilizarse en la producción de jamones tipo serrano, principalmente con piernas de más de $10.00 \mathrm{~kg}$ provenientes de animales de $90.00 \mathrm{~kg}$ o más; este peso se asocia a la máxima ganancia cuando el precio de venta del animal es de $\$ 60$ por $\mathrm{kg}$. Así, los productos obtenidos le agregan mayor valor económico a la carne del CPM, mismos que se han considerado como oferta en el mercado gourmet, ventaja que se obtiene al integrarse la correspondiente cadena productiva y de valor.

Los otros escenarios de rentabilidad de la etapa IV, como se observa en la Tabla 2, consideran precios de venta del animal que varían de $\$ 35.00$ a $\$ 45.00$ por kg y los pesos óptimos del mismo son de 52.00 a $75.00 \mathrm{~kg}$, dichos escenarios son considerados cuando los animales son alimentados con $100 \%$ de alimento balanceado y la acumulación de grasa subcutánea e intramuscular va en aumento con el peso del animal, características deseables para la industria de elaboración de productos curados. En la etapa de engorda del animal, el costo por kilogramo de peso vivo obtenido resulta superior al registrado en los sistemas de producción con animales mejorados, pero éste puede ser reducido por medio del uso de forrajes verdes de plantas arbustivas o arbóreas (Sierra et al. 2016); estrategia alimenticia que se justifica debido al lento crecimiento del CPM.

Por otra parte, y con relación al manejo alimen- 
ticio del CPM, se ha demostrado la factibilidad de la sustitución de una parte de los insumos comerciales utilizados en la elaboración de alimento balanceado, como la inclusión de harina de hojas de morera; práctica que reduce hasta en un $18.5 \%$ el precio por kilogramo del alimento balanceado (OsortoHernández et al.2007). Los óptimos económicos correspondientes se alcanzan con animales más pesados, con rango de 46.00 a $86.00 \mathrm{~kg}$. Una ventaja de la sustitución de insumos alimenticios es la oferta de animales menores de $50.00 \mathrm{~kg}$ para el segmento de elaboración de platillos regionales, como lechón al horno, cochinita pibil u otros donde las características organolépticas de la carne marcan la diferencia en sabor con respecto al que ofrecen los cerdos mejorados, debido a su menor contenido de grasa (Linares et al. 2011). El menor costo por kilogramo de alimento favorece que se envíe al mercado animal con pesos óptimos más altos, pero en ambos casos el manejo de la alimentación satisface la demanda de materia prima para la elaboración de diversos productos derivados de la carne del CPM, desde el envasado de canales pequeñas hasta la oferta de animales propios para la elaboración de jamones tipo serrano.

La ganancia diaria de peso (gdp) del CPM es superior a lo reportado por Cetz et al. (2005) quienes evaluaron la gdp con restricción de alimento balanceado (PC 16\%) más verduras, hortalizas y restos de comida, reportando animales de $9.60 \mathrm{~kg}$ a la edad de 120 días con ganancias de 0.07 hasta 0.08 $\mathrm{kg} \mathrm{d}^{-1}$; en el presente se registró un peso similar en la etapa I, pero a una edad más temprana (49 días) con ganancias superiores al citado $\left(0.24 \mathrm{~kg}\right.$ día $\left.{ }^{-1}\right)$. Pero los parámetros encontrados resultaron más bajos en comparación con lo reportado por Zhan et al. (2019) para cerdos (Duroc $x$ Landrace $x$ Yorkshire) con peso inicial de 64.30 y final de $108.70 \mathrm{~kg}$, alimentados con una dieta convencional de $15 \%$ de $\mathrm{PC}$, la ganancia obtenida fue de $0.99 \mathrm{~kg}$ día $^{-1}$, superior al encontrado en la etapa IV; mientras que Camacho et al. (2008) al evaluar las gdp de CPM $y$ cerdos Landrace $x$ Yorkshire encontraron un comportamiento similar. Los resultados sugieren que la correcta cantidad y calidad de proteína en la dieta op- timiza la eficiencia y maximiza la producción en cerdos en crecimiento (Whittemore et al. 2001); mientras que estudios realizados por Conde-Aguilera et al. (2011) en cerdo ibérico indican que además de la alimentación y etapa fisiológica, la genética juega un papel importante en el comportamiento productivo. El CPM al ser un animal obeso y de lento crecimiento al igual que el ibérico, sus requerimientos nutricionales difieren de los cerdos mejorados debido a su poca capacidad para retener nitrógeno $(\mathrm{N})$ (Lemus et al. 2008, Santos-Ricalde et al. 2011), lo que se demuestra por la diferencia en la eficiencia alimenticia. Un aspecto relevante en la engorda de este cerdo criollo es el costo de la alimentación, debido a que ante la propuesta de integrarlo a un sistema de producción semitecnificado con fin empresarial, donde el insumo principal es el alimento balanceado, su eficiencia productiva y la variación en el costo de producción son de interés en la determinación del óptimo económico en la engorda. La alimentación con alimento 100\% balanceado se tiene la ganancia de peso constante a lo largo del período de engorda, lo que indica la importancia de utilizar la alimentación alternativa a partir de la etapa III, con el fin de no perder la oportunidad de mayores ganancias derivadas de la disminución de los costos medios. Por lo que a pesar del precio de venta de destete, es rentable continuar con el proceso de engorda siempre y cuando se realicen análisis económicos de los costos de producción y precios de venta con los que se pueda determinar el momento adecuado para el envío de los animales al mercado.

Para la crianza del CPM en el ámbito empresarial de manera sustentable, se requiere analizar diferentes escenarios de precios de venta del animal en pie (Tabla 3), ya que es de importancia para el porcicultor determinar el peso y la edad óptima de venta, para determinar el precio que más le conviene negociar con los participantes del segundo eslabón productivo. Aunado a lo anterior, también es conveniente el análisis de diferentes alternativas de sustitución de granos en la elaboración del alimento balanceado, ya que el costo de este insumo es relevante en la rentabilidad de la empresa. 


\section{CONCLUSIONES}

La eficiencia alimenticia del CPM fue mayor en las etapas I y II de engorda, pero en general el valor de los parámetros de su comportamiento productivo representan poco menos de la mitad del registrado para el cerdo mejorado, a pesar de que se utilizó alimento balanceado. A medida que el precio de venta del animal se incrementa, su peso óptimo de mercado o de máxima ganancia económica varía, no así con respecto al precio del alimento, cuya relación fue inversa; lo primero ocurre según la cantidad y calidad de las canales que demande la agroindustria procesadora de jamones y productos curados de alta cali- dad o de platillos regionales. Por lo que la integración de las granjas productoras de CPM como parte inicial de la cadena productiva, favorece la obtención de mayor nivel de rentabilidad y la conservación de dicho recurso zoogenético.

\section{AGRADECIMIENTOS}

Al Tecnológico Nacional de México por el financiamiento otorgado mediante el proyecto "Curva de crecimiento en hembras de cerdo pelón mexicano". Clave 6310.1.

\section{LITERATURA CITADA}

Camacho-Rea C, Gutiérrez CR, Arechavaleta-Velasco MG, Díaz CL.D, Arechavaleta VFJ, Martínez RR, Lemus FC, Alonso MRA (2008) Características endocrinas, moleculares y de parámetros de crecimiento asociados a la obesidad del cerdo Pelón Mexicano. Revista Mexicana de Ciencias Pecuarias 46: 345-357.

Cetz F, Irigoyen J, Sierra A, Medrano A (2005) Caracterización zootécnica del cerdo pelón mexicano explotado en un centro de conservación genética. Veterinaria 40: 41-44.

Conde-Aguilera JA, Aguinaga MA, Aguilera JF, Nieto R (2011) Nutrient and energy retention in weaned iberian piglets fed diets with different protein concentrations. Journal of Animal Science 89: 754-763.

Delgado GL, Gómez CS, Rubio LMS, Capella VS, Méndez MD, Labastida RC (2002a) Fatty acid and triglyceride profiles of intramuscular and subcutaneous fat from fresh and dry-cured hams from Hairless Mexican pigs. Meat science 61: 61-65.

Delgado GL, Gómez CS, Rubio LMS, Iturbe CHF, Méndez MD (2002b) Evolution in the shoulder composition of hairless Mexican pigs throughout the curing and drying processes. Meat Science 61: 341-346.

García E (1981) Modificaciones al sistema de clasificación climática de Köppen. Para adaptarlo a las condiciones de la República Mexicana. Instituto de Geografía, Universidad Nacional Autónoma de México. México. 217p

Grunert KG, Sonntag WI, Glanz-Chanos V, Forum S (2018) Consumer interest in environmental impact, safety, health and animal welfare aspects of modern pig production: Results of a cross-national choice experiment. Meat Science 137: 123-129.

Kay RD (1986) Administración agrícola y ganadera: planeación, control e implementación. Compañía Editorial Continental. Primera edición. Texas, USA. 221p.

Lemus C, Hernández-Ballesteros JA, Navarrete R, Rodríguez JG, de la Barrera JLJ (2008) Algunos estudios de manejo de cerdos pelón mexicano durante el crecimiento. Revista Computadorizada de Producción Porcina 15: 158-162.

Linares V, Linares L, Mendoza G (2011) Caracterización etnozootécnica y potencial carnicero de Sus scrofa “cerdo criollo"en Latinoamérica. Scientia Agropecuaria 2: 97-110. 
Lopez-Bote C, Fructuoso G, Mateos GG (2000) sistemas de producción porcina y calidad de la carne. El cerdo Ibérico. XVI. Curso de especialización FEDNA. España. http://fundacionfedna.org/sites/default/files/00CAP 5.pdf. Fecha de consulta: 3 de mayo de 2019.

Méndez-Medina RD, Becerríl-Herrera M, Rubio-Lozano MDLS, Delgado-Suárez EJ (2002) Características de la canal del cerdo Pelón Mexicano, procedente de Mizantla, Veracruz, México. Veterinaria México 33: 27-37.

Mie A, Andersen HR, Gunnarsson S, Kahl J, Kesse-Guyot E, Rembiałkowska E, Quaglio G, Grandjean P (2017) Human health implications of organic food and organic agriculture: a comprehensive review. Environmental Health 16: 111-133.

Nieto R, Lara L, Barea R, Garcia-Valverde R, Conde-Aguilera JA, Aguilera JF (2014) An analysis of the composition of gain and growth of primal cuts of lberian pigs of 10 to $150 \mathrm{~kg}$ body weight as affected by the level of feeding and dietary protein concentration. Spanish Journal of Agricultural Research 12: 1082-1093.

NRC (2010) Guide for the care and use of laboratory animals. National Research Council. National Academic Press, Washington DC. 247p.

NRC (2012) Nutrient Requirement of Swine. National Research Council. National Academic Press, Washington DC. $210 p$.

Oelke CA, Bernardi ML, Nunes PR, Weber NC, Veit FC, Leal-Ribeiro AM (2018) Physiological and behavioural response of sows fed with different levels of dietary fiber during gestation. Journal of Veterinary Behavior 28: 54-57.

Osorto-Hernandéz WA, Lara Lara PE, Magaña Magaña MA, Sierra Vásquez AC, Sanginés García JR (2007) Morera (Morus alba) fresca o en forma de harina en la alimentación de cerdos en crecimiento y engorda. Revista Cubana de Ciencia Agrícola 41: 49-63.

Peña Y, Nieto-Alemán PA, Díaz-Rodríguez F (2008) Cadenas de valor: un enfoque para las agrocadenas. Equidad y Desarrollo 9: 77-85.

Pindyck R, Rubinfeld D (2009) Microeconomía. Séptima edición. Pearson Educación. Madrid, España. 850p.

Pugliese C, Sirtori F (2012) Quality of meat and meat products produced from southern European pig breeds. Meat Science 90: 511-518.

Rebollar SR, Tenorio GG, Matínez JH, Rubio RR, Razo DJG, Nova FA (2007) Determinación del óptimo técnico y económico en una granja porcícola en Temascaltepec, Estado de México. Ciencia Ergo Sum 14: 255-262

Régnier C, Bocage B, Archimède H, Noblet J, Renaudeau D (2013) Digestive utilization of tropical foliages of cassava, sweet potatoes, wild cocoyam and erythrina in Creole growing pigs. Animal Feed Science and Technology 180: 44-54.

Renaudeau D, Mourot J (2007) A comparison of carcass and meat quality characteristics of Creole and Large White pigs slaughtered at $90 \mathrm{~kg} \mathrm{BW}$. Meat Science 76: 165-171.

Santos-Ricalde R, Trejo-Lizama W, Osorto-Hernández W (2011) Rendimiento de la canal y desarrollo de los órganos torácicos y abdominales de los 25 a los $45 \mathrm{~kg}$ en cerdos criollos pelones. Revista Científica XXI: 396-402.

Serra X, Gil F, Pérez-Enciso M, Oliver MA, Vázquez JM, Gisperta M, Dias I, Moreno F, Latorre R, Noguera JL (1998) A comparison of carcass, meat quality and histochemical characteristics of lberian (Guadyerbas line) and Landrace pigs. Livestock Production Science 56: 215-223. 
Sierra VAC, Ortiz OJR, Bojórquez CJC, Canul SMA, Tamayo CJR, Rodríguez PJC, Sanginés GJR, Magaña MMA, Montes PRC, Segura CJC (2016) Conservación y uso sustentable del cerdo pelón en Yucatán. Quehacer Científico en Chiapas 11: 13-28.

Su Y, Long Y, Liao X, Ai, H, Zhang Z, Yang B,Xiao S, Tang J, Xin W, Huang L, Ren J, Ding N (2014) Detection of genomic signatures for pig hairlessness using high-density SNP data. Frontiers of Agricultural Science and Engineering 1: 307-313.

Whittemore CT, Green DM, Knap PW (2001) Technical review of the energy and protein requirements of growing pigs: energy. Animal Science Glasgow 73: 199-216.

Zhang T, Si B, Tu Y, Cui K, Zhou C (2019) Effect of including different levels of moringa (Moringa oleifera) leaf meal in the diet of finishing pigs: Performance, pork quality, fatty acid composition, and amino acid profile. Czech Journal of Animal Science 64: 141-149. 